
impressive experimental results. However, since this method resamples a data frame of many OFDM symbols with a single factor, it does not work well when the velocity changes roughly over OFDM symbols. In addition, this method requires storing an entire data frame of many OFDM symbols to estimate the time expansion/compression. Most importantly, the Doppler effect does not always manifest itself significantly in time expansion/compression, so high accuracy estimation and time scale are not easy. For example, a moving speed of $1 \mathrm{~m} / \mathrm{s}$ causes a Doppler shift of $16 \mathrm{~Hz}$ equivalent to $16 \%$ of subcarrier space $\left(f_{0}=93.75 \mathrm{~Hz}\right)$, but the expansion/ compression is only 1 (sample/an OFDM symbol).

Our proposal overcomes the drawbacks of the resampling based method. Our ideas are using continual pilots (CP) to track Doppler shift over each OFDM symbol and compensating Doppler symbol-by-symbol in the frequency domain rather than resampling in time domain. Though velocity changed roughly during pulling/pushing transmitters in our experiments, our method tracked and compensated Doppler shift well. However, the maximum Doppler shift can be estimated by using $\mathrm{CP}$ as $1 / 3$ of subcarrier space in our case. To boost the estimation range, we propose a solution through monitoring the drift of Power Delay Profile (PDF) over OFDM symbols. The Doppler effect causes time expansion/compression; thus, when the FFT window is fixed at receivers $\mathrm{PDF}$ will be drifted over time. By measuring the drift amount of PDF, Doppler shift can be roughly estimated. Following the rough estimation is a fine Doppler shift estimation using continual pilots.

Then, the final estimation of Doppler shift is used in two stages to remove impacts of the Doppler shift. In the first stage, a simple phase derotation is performed before FFT. In the second stage, with an assumption that all delay paths has a similar Doppler rate, we separate impacts of Doppler shift from multipath channel and derive an ICI matrix which represents the position dependent Doppler shift. The ICI matrix is constructed using only the final estimation of Doppler shift and does not require estimation of channel transfer function yet. Thus, ICI is removed before channel estimation that is different from conventional ICI matrix in [1-5]. Overall, simulation and experiments results show that our method outperforms the typical resampling method [11].

The rest of this paper is organized as follows. Section 2 presents signal model. The proposed system is presented in Section 3. Section 4 presents simulation and experimental results. Finally, conclusion is given Section 5 .

\section{Signal Model}

The transmitted signal of OFDM symbol $m$ can be written as

$$
\begin{array}{r}
S(t)=\operatorname{Re}\left\{\sum_{k=-N}^{N} D(k, m) e^{j 2 \pi\left(f_{c}+k f_{0}\right)\left(t+m T_{\mathrm{sb}}+T_{\mathrm{GI}}\right)}\right\} \\
\text { with } 0 \leq t \leq T_{0}
\end{array}
$$

$T_{\mathrm{sb}}=T_{0}+T_{\mathrm{GI}}$
Here, $T_{0}$ and $T_{\mathrm{sb}}$ is the symbol length of an OFDM symbol excluded and included guard interval, respectively, and $T_{\mathrm{GI}}$ is the guard interval length. Totally, $(2 N+1)$ subcarriers are utilized to carry information data. $f_{c}$ and $f_{0}$ are carrier frequency and subcarrier space, respectively. $D(k, m)$ denotes data at subcarrier $k$ of OFDM symbol $m$. The relative moving between transmitters and receivers caused a Doppler rate as

$$
\Delta(t)=\frac{v(t)}{C}
$$

Here, $v(t)$ is the relative moving speed between a transmitter and receivers, and $C \approx 1500 \mathrm{~m} / \mathrm{s}$ is propagation speed of sound in the water. The time varying Doppler rate $\Delta(t)$ is assumed constant within two successive OFDM symbols but changes over OFDM symbols. In addition, we assumed that there are $L$ multipaths; each path has a gain of $r_{i}$ and a delay of $\tau_{i}$, and all paths have a similar Doppler rate $\Delta$. Thus, the received pass-band signal is written as follows:

$$
R_{P B}(t)=\sum_{i=1}^{L} S\left((1+\Delta)\left(t-\tau_{i}\right)\right) .
$$

In the time domain, the Doppler effect manifests itself in time expansion/compression that is $\Delta N_{s} T_{s}$ over an OFDM symbol. Here, $N_{s}$ is number of discrete samples of an OFDM symbol, and $T_{s}$ is a sampling period. A straightforward idea is resampling the distorted signal to compensate the Doppler effect [6-13]. Different from those methods, we analyze and compensate impacts of the Doppler in frequency domain. After downconversion, the received signal at baseband can be written as

$$
\begin{aligned}
& R_{B B}(t)=\{\sum_{k=-N}^{N} H(k, m) D(k, m) \underbrace{e^{j 2 \pi k f_{0}(1+\Delta) t}}_{\begin{array}{c}
\text { non-uniform } \\
\text { Doppler shift }
\end{array}}\} \\
& \quad \underbrace{e^{j 2 \pi f_{c} \Delta t} \beta(m),}_{\begin{array}{c}
\text { common } \\
\text { phase rotation }
\end{array}} \\
& \beta(m)=e^{j 2 \pi f_{c} \Delta\left(m T_{\mathrm{sb}}+T_{\mathrm{GI}}\right)}, \\
& H(k, m)=\sum_{i=1}^{L} r_{i} e^{-j 2 \pi\left(f_{c}+k f_{0}\right)(1+\Delta) \tau_{i}} e^{j 2 \pi k f_{0} \Delta\left(m T_{\mathrm{sb}}+T_{\mathrm{GI}}\right)} .
\end{aligned}
$$

As in (4), all subcarriers experience a common frequency offset as $e^{j 2 \pi f_{c} \Delta t}$. Each subcarrier experiences a different frequency offset of as $e^{j 2 \pi k f_{0} \Delta t}$ depending on the position of the subcarrier. This is called position-dependent frequency offset, or the so-called nonuniform Doppler shift in [12]. The position-dependent frequency offset significantly degrades performance of high modulation such as 64QAM. In our case, a moving speed of $1 \mathrm{~m} / \mathrm{s}$ causes the common Doppler shift of $16 \mathrm{~Hz}$ which is equal to $16 \%$ of subcarrier space. In addition, the edge subcarriers corresponding to $k= \pm 40$ suffers a frequency offset of $\pm 2.5 \mathrm{~Hz}$ which is equivalent to $2.5 \%$ of subcarrier space. The central subcarrier $k=0$ does not experience this kind of frequency offset. Therefore, we must 


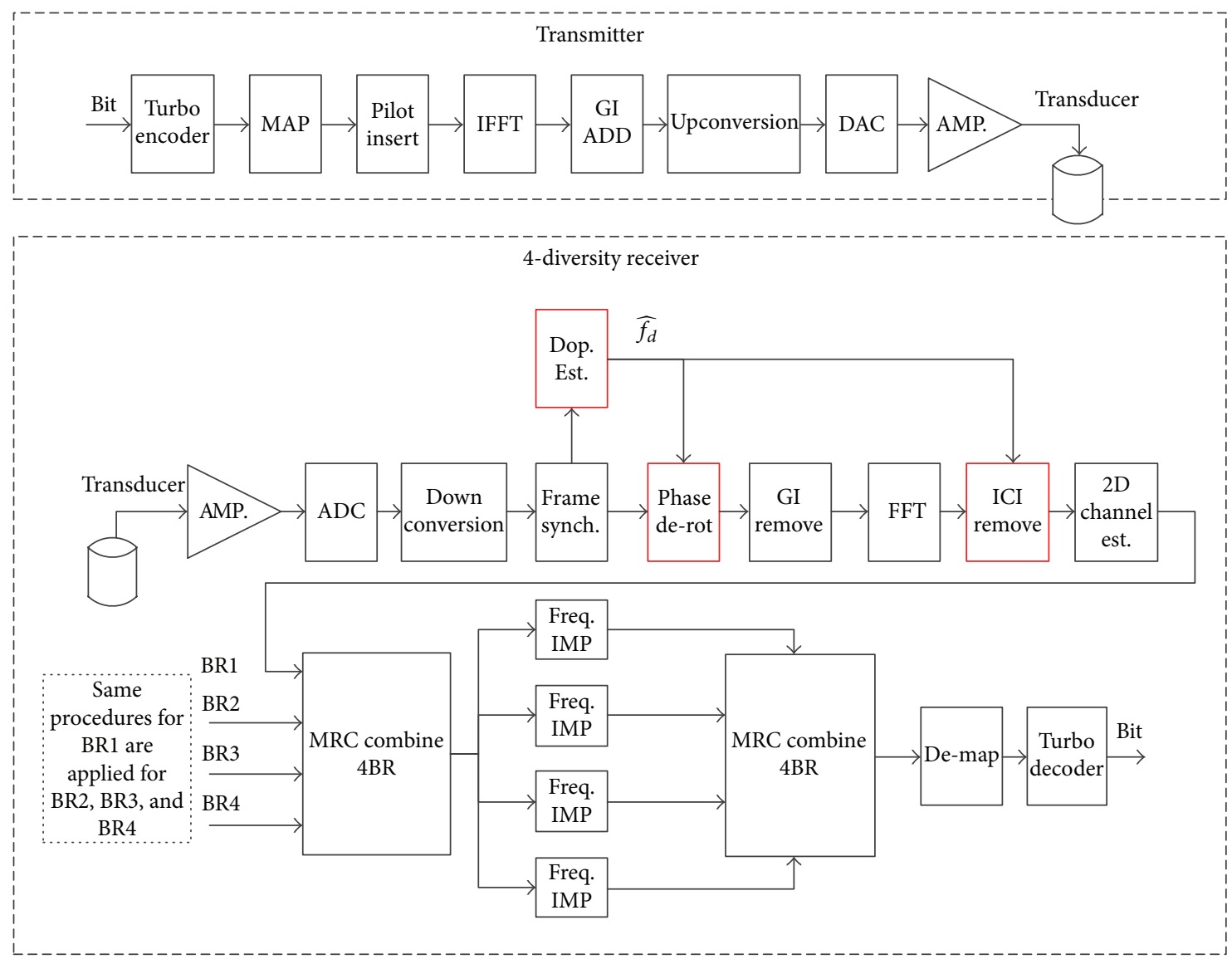

FIgURE 1: Proposed transceiver architecture.

take position-dependent frequency offset into account. Estimation of the Doppler rate and compensation of its impacts are presented in the next section.

\section{The Proposed System}

Transducers attached to the bottom of the ship swing due to ocean wave and the robot at the sea bottom also move in a complicated trajectory. Therefore, time varying Doppler shift changes over OFDM symbols. To support such a case, the highlight of our system are time varying Doppler shift estimation, and symbol-by-symbol nonuniform Doppler compensation. In addition, impulsive noise cancellation [14-16] also is incorporated in our system. System parameters are shown in Table 1 and the overall system architecture is shown in Figure 1.

3.1. Estimation of Time Varying Doppler Shift. In this section we present a time varying Doppler shift estimation by monitoring the drift of PDF in conjunction with using continual pilots. Using continual pilots can estimate Doppler shift accurately; however, as explained later the maximum Doppler shift can be estimated is limited by $f_{0} / 2\left(1+T_{\mathrm{GI}} / T_{0}\right)$, which is $f_{0} / 3$ in our system. To boost the estimation range, we propose a rough Doppler estimation through monitoring the drift of
TABLE 1: Our system parameters.

\begin{tabular}{|c|c|c|}
\hline Parameters & \multicolumn{2}{|c|}{ Value } \\
\hline TX-RX elements & $1 \mathrm{TX} / 4$ & transducer \\
\hline Sampling frequency & \multicolumn{2}{|c|}{$96000 \mathrm{~Hz}$} \\
\hline TX center frequency & \multicolumn{2}{|c|}{$24000 \mathrm{~Hz}$} \\
\hline Band width & \multicolumn{2}{|c|}{$7593 \mathrm{~Hz}$} \\
\hline FFT size & \multicolumn{2}{|c|}{1024} \\
\hline OFDM symbol length $T_{0}$ excluded GI & \multicolumn{2}{|c|}{$10.67 \mathrm{~ms}$} \\
\hline GI length $T_{\mathrm{GI}}$ & \multicolumn{2}{|c|}{$0.5 T_{0}$} \\
\hline Sub carrier spacing & \multicolumn{2}{|c|}{$93.75 \mathrm{~Hz}$} \\
\hline Number of subcarrier & \multicolumn{2}{|c|}{81} \\
\hline Modulation & 16QAM & 64QAM \\
\hline Turbo code rate & $1 / 2$ & $1 / 3$ \\
\hline Space among transducers at RX side & \multicolumn{2}{|c|}{$10 \mathrm{~cm}$} \\
\hline
\end{tabular}

PDF due to Doppler-induced expansion/compression. The signal processing of Doppler estimation is shown in Figure 2. 


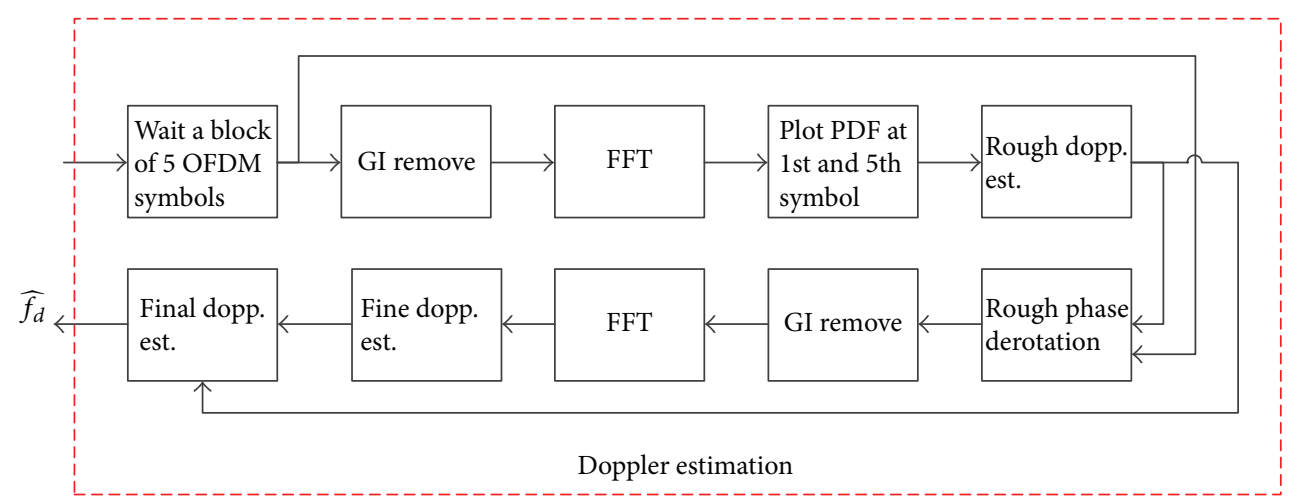

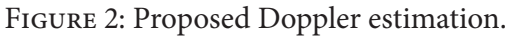

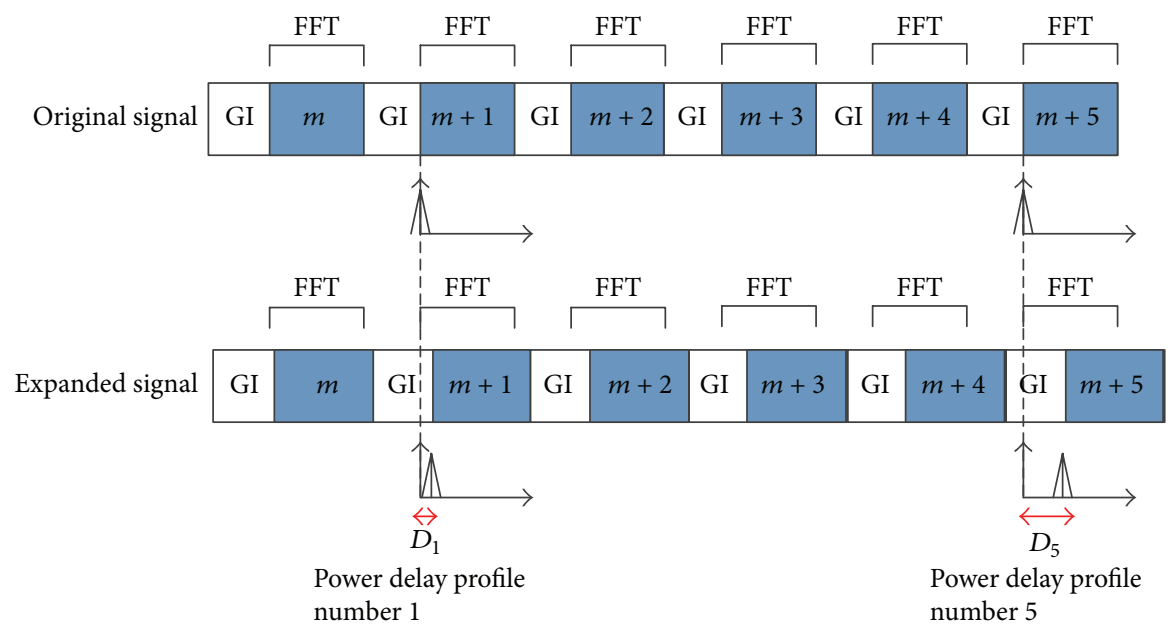

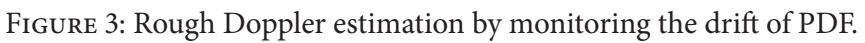

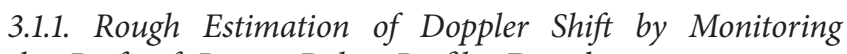

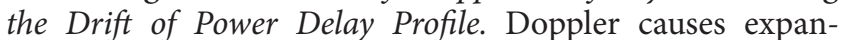

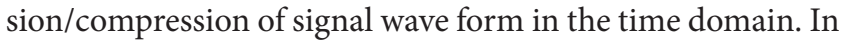

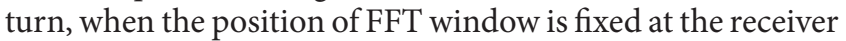

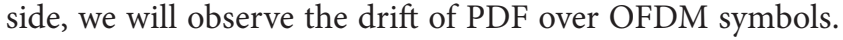

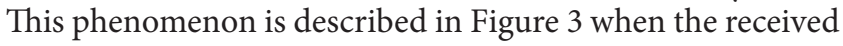

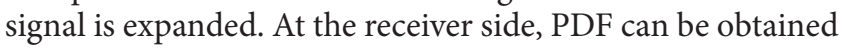

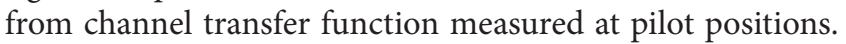

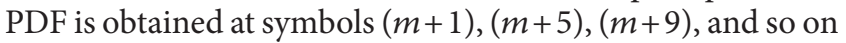

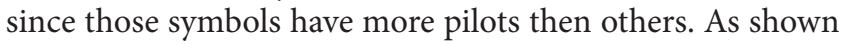

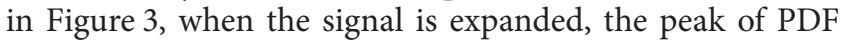

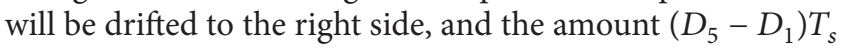

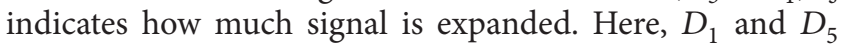

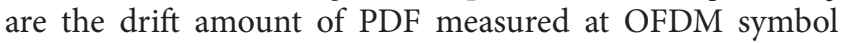

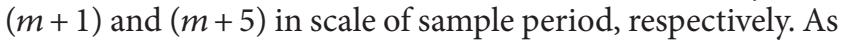

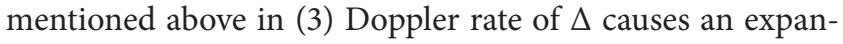

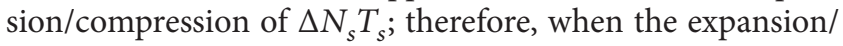

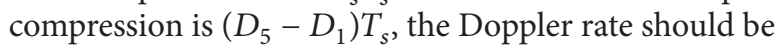

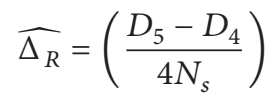

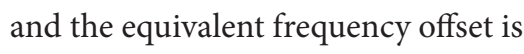

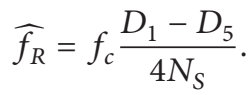

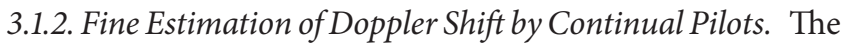

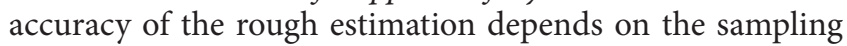

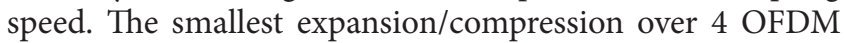

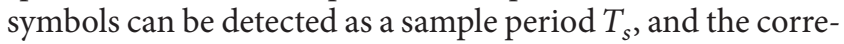

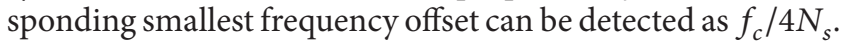

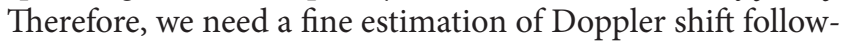

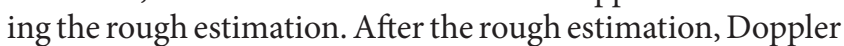

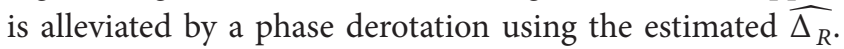

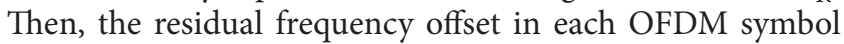

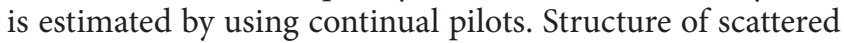

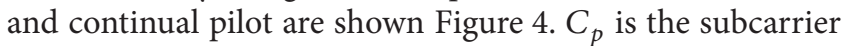

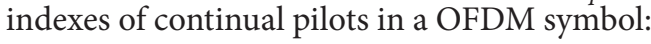

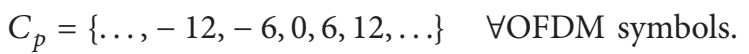




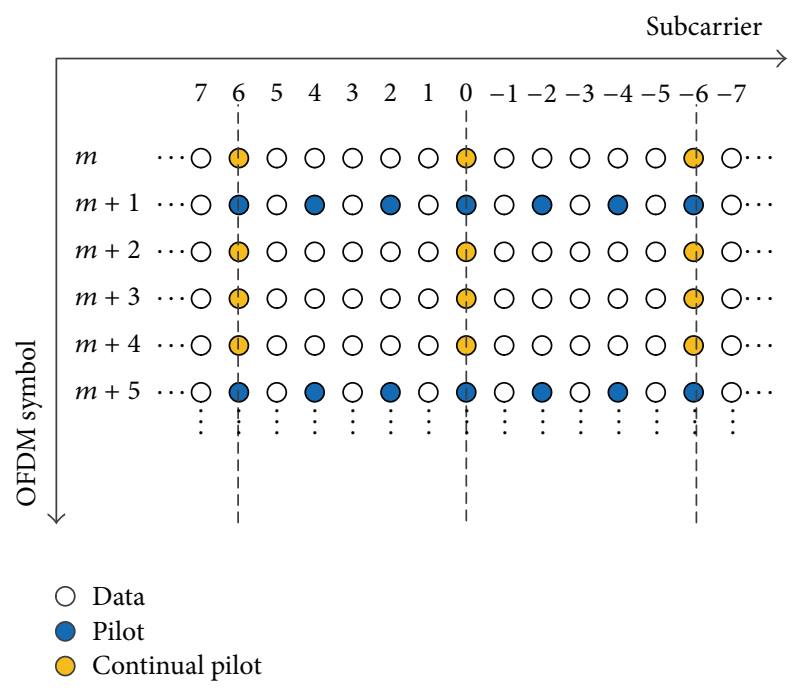

Figure 4: Pilot structure.
After the phase derotation, the baseband signal in (4) is rewritten as

$$
\begin{aligned}
& R_{B B}^{(1)}(t)=\{\sum_{k=-N}^{N} H(k, m) D(k, m) \underbrace{e^{j 2 \pi k f_{0}(1+\Delta) t}}_{\begin{array}{c}
\text { non-uniform } \\
\text { Doppler shift }
\end{array}}\} \\
& \cdot \underbrace{e^{j 2 \pi f_{c}\left(\Delta-\widehat{\Delta_{R}}\right) t} \gamma(m)}_{\begin{array}{c}
\text { common } \\
\text { phase rotation }
\end{array}}, \\
& \gamma(m)=e^{j 2 \pi f_{c}\left(\Delta-\widehat{\Delta_{R}}\right)\left(m T_{\mathrm{sb}}+T_{\mathrm{GI}}\right)}, \\
& f_{e}=f_{c}\left(\Delta-\widehat{\Delta_{R}}\right) .
\end{aligned}
$$

After performing FFT demodulation on the received signal $R_{B B}^{(1)}(t)$, the received signal at subcarrier $k$ of symbol $m$ is written as

$$
\begin{aligned}
Y(k, m)= & H(k, m) D(k, m) \gamma(m) e^{j \pi\left(k \Delta+f_{e} / f_{0}\right)} \\
& +\underbrace{\sum_{\substack{l=-N \\
l \neq k}}^{N} H(l, m) D(l, m) \gamma(m) \operatorname{sinc}\left(\pi\left(l-k+l \Delta+\frac{f_{e}}{f_{0}}\right)\right) e^{j \pi\left(l-k+l \Delta+f_{e} / f_{0}\right)},}_{\text {ICI }} \\
Y(k, m) \approx & H(k, m) D(k, m) \gamma(m) e^{j \pi\left(k \Delta+f_{e} / f_{0}\right)} .
\end{aligned}
$$

Similarity, the received signal at subcarrier $k$ of OFDM symbol $(m+1)$ is calculated as

$$
\begin{aligned}
& Y(k, m+1) \\
& \quad \approx H(k, m+1) D(k, m+1) \gamma(m+1) e^{j \pi\left(k \Delta+f_{e} / f_{0}\right)} .
\end{aligned}
$$

To estimate the residual Doppler shift $f_{e}$, we consider the phase difference in the channel transfer function $H(k, m)$ and $H(k, m+1)$. We denote

$$
\begin{aligned}
W(k, m) & =\frac{Y(k, m)}{D(k, m)}, \quad k \in C_{p}, \\
W(k, m+1) & =\frac{Y(k, m+1)}{D(k, m+1)}, \quad k \in C_{p} .
\end{aligned}
$$

It is worth noting that

$$
\begin{aligned}
\operatorname{conj} & (H(k, m)) H(k, m+1) \\
= & |\operatorname{conj}(H(k, m)) H(k, m+1)| e^{j 2 \pi k f_{0} \Delta T_{\mathrm{sb}},}
\end{aligned}
$$

$$
\operatorname{conj}(\gamma(m)) \gamma(m+1)=e^{j 2 \pi f_{c}\left(\Delta-\widehat{\Delta_{R}}\right) T_{\mathrm{sb}}}
$$

With $k \in S_{p}, D(k, m)$ and $D(k, m+1)$ are preknown continual pilots at the receiver side. From (13)-(15) the residual Doppler shift can be estimated as

$$
\begin{aligned}
\widehat{f_{e}}= & \frac{f_{0}}{2 \pi\left(1+T_{\mathrm{GI}} / T_{0}\right)} \\
& \cdot L\left\{\sum_{k \in C_{p}} \operatorname{conj}(W(k, m)) W(k, m+1)\right\} .
\end{aligned}
$$

Due to the angle $-\pi \leq \angle\left\{\sum_{k \in C_{p}} \operatorname{conj}(W(k, m)) W(k, m+\right.$ $1)\} \leq \pi$, the maximum Doppler shift can be estimated as $f_{0} / 2\left(1+T_{\mathrm{Gi}} / T_{0}\right)$. It is noted that the continual pilots are placed symmetrically over the central subcarrier as in (9). Therefore, the element $e^{j 2 \pi k f_{0} \Delta}$ will be self-called in (17). The total frequency offset $\widehat{f}_{d}$ is sum of rough and fine estimate values. The estimate frequency offset will be used for phase derotation, and for constructing the ICI matrix in the next section: 


$$
\begin{aligned}
\widehat{f_{d}} & =\widehat{f_{R}}+\widehat{f_{e}}, \\
\widehat{\Delta} & =\frac{\widehat{f_{d}}}{f_{c}} .
\end{aligned}
$$

\subsection{Symbol-by-Symbol Doppler Compensation}

3.2.1. Phase Derotation. We rewrite the received after downconversion from (4) as

$$
\begin{aligned}
R_{B B}(t)= & \{\sum_{k=-N}^{N} H(k, m) D(k, m) \underbrace{e^{j 2 \pi k f_{0}(1+\Delta) t}}_{\begin{array}{c}
\text { non-uniform } \\
\text { Doppler shift }
\end{array}}\} \\
& \cdot \underbrace{e^{j 2 \pi f_{c} \Delta t} \beta(m)}_{\begin{array}{c}
\text { common } \\
\text { phase rotation }
\end{array}} .
\end{aligned}
$$

$$
\left[\begin{array}{c}
Y(-N, m) \\
Y(-N+1, m) \\
\vdots \\
Y(N+1, m) \\
Y(N, m)
\end{array}\right]
$$$$
=\underbrace{\left[\begin{array}{ccccc}
I(-N,-N) & I(-N,-N+1) & \cdots & I(-N, N-1) & I(-N, N) \\
I(-N+1,-N) & I(-N+1,-N+1) & \cdots & I(-N+1, N-1) & I(-N+1, N) \\
\vdots & \vdots & \ddots & \vdots & \vdots \\
I(N-1,-N) & I(N-1,-N+1) & \cdots & I(N-1, N-1) & I(N-1, N) \\
I(N,-N) & I(N,-N+1) & \cdots & I(N, N-1) & I(N, N)
\end{array}\right]}_{\text {ICI matrix representing non-uniform Doppler }} \underbrace{\left[\begin{array}{c}
H(-N, m) D(-N, m) \\
H(N+1, m) D(-N+1, m) \\
H(N-1, m) D(N-1, m) \\
H(N, m) D(N, m)
\end{array}\right]}_{\text {Channel Transfer Function }},
$$$$
I(k, l)= \begin{cases}e^{j \pi \Delta k} & \text { if } k=l \\ \operatorname{sinc}(\pi(l-k+l \Delta)) e^{j \pi(l-k+l \Delta)} & \text { if } k \neq l\end{cases}
$$

$I(k, l)$ represents the amplitude of intercarrier interference from subcarrier $l$ to subcarrier $k$. It is noted that $I(k, l)$ not just depends on Doppler rate $\Delta$, and distance $(l-k)$ between two subcarriers, but also depends on position of subcarrier $l$. In another word, edge subcarriers suffer severe ICI compared to center subcarriers. Using the estimation of Doppler rate $\widehat{\Delta}$, the ICI matrix can be determined as

$$
\widehat{I}(k, l)= \begin{cases}e^{j \pi \widehat{\Delta} k} & \text { if } k=l \\ \operatorname{sinc}(\pi(l-k+l \widehat{\Delta})) e^{j \pi(l-k+l \widehat{\Delta})} & \text { if } k \neq l\end{cases}
$$

The first stage is compensating the common phase derotation using the estimation of Doppler rate $\widehat{\Delta}$. Since the estimation of Doppler rate $\widehat{\Delta} \approx \Delta$, after phase derotation we get

$$
R_{B B}^{(2)}(t)=\sum_{k=-N}^{N} H(k, m) D(k, m) \underbrace{e^{j 2 \pi k f_{0}(1+\Delta) t}}_{\begin{array}{c}
\text { non-uniform } \\
\text { Doppler shift }
\end{array}} .
$$

3.2.2. ICI Cancellation for Nonuniform Doppler. After performing FFT demodulation on $R_{B B}^{(2)}(t)$, the received data at subcarrier $k$ of OFDM symbol $n$ is calculated as

$$
\begin{gathered}
Y(k, m)=H(k, m) D(k, m) e^{j \pi \Delta k}+\sum_{\substack{l=-N \\
l \neq k}}^{N} H(l, m) \\
\cdot D(k, m) \operatorname{sinc}(\pi(l-k+l \Delta)) e^{j \pi(l-k+l \Delta)} .
\end{gathered}
$$

In matrix form,
ICI is removed before channel estimation as

$$
\left[Y_{I C I}\right]=\frac{[Y]}{[\widehat{I}]}
$$

Here,

$$
Y_{\mathrm{ICI}}(k, m)=H(k, m) D(k, m)
$$

After ICI is removed from received signal, the free-ICI signal $Y_{\text {ICI }}(k, m)$ is fed into the estimation block and the remained channel transfer function $H(k, m)$ can be easily estimated by 
using pilots, and 1-Taps equalizer is sufficient to recover data $D(k, m)$ :

$$
\widehat{D}(k, m)=\frac{Y_{\text {ICI }}(k, m)}{\widehat{H}(k, m)} .
$$

Here, $\widehat{H}(k, m)$ is estimation of channel transfer function provided by the channel estimation block.

3.3. Reduction of Computational Complexity. The resampling based method requires arbitrary rate resampling. Lagrange interpolation can be applied to perform arbitrary rate resampling, and coefficients of the Lagrange polynomial can be computed through finding inversion of the Vandermonde matrix. On the other hand, our rough estimation and the fine estimation have complexity of $\mathrm{O}(n \log n)$ since these procedures utilize FFT to measure the PDF at OFDM symbols $m, m+4, m+8$, and so on, and FFT demodulation to compute $Y(k, m)$ and $Y(k, m+1)$ in (12) and (13). However, the ICI cancellation requires finding the inversion of the ICI matrix, so this procedure has complexity of $O\left(n^{3}\right)$. To avoid matrix inversion, we can apply the Jacobi iterative method for the big ICI matrix equation (22), and the complexity is reduced to $O\left(n^{2}\right)$. By applying the Jacobi iterative method for (22), the initial value of $\left[Y_{\text {ICI }}\right]$ is computed as

$$
\left[Y_{\mathrm{ICI}}^{(0)}\right]=\frac{[Y]}{\operatorname{diag}(\widehat{I})} .
$$

Then, at the second iteration,

$$
\left[Y_{\mathrm{ICI}}^{(1)}\right]=\frac{[Y]-\{[\widehat{I}]-\operatorname{diag}(\widehat{I})\}\left[Y_{I C I}^{(0)}\right]}{\operatorname{diag}(\widehat{I})}
$$

$\left[Y_{I C I}^{(1)}\right]$ is fed into the channel estimation and then 1-Taps equalizer to recover the data $[D]$.

\section{Simulation and Experimental Results}

In this section, simulation and experimental results confirm that our proposal yields a better performance than the resampling based method. It is worth noting that all Bit Error Rate (BER) shown in the following is before Turbo decoding. After Turbo decoding, our system achieves free error in both simulation and experiments.

\subsection{Simulation Result}

4.1.1. Performance of the ICI Cancellation for Nonuniform Doppler. The channel condition is shown in Table 2, and all paths have a similar Doppler rate $\Delta$. In Figure 5, "wo" means without Doppler compensation. " $w d$ " means Doppler compensating using only the diagonal of the ICI matrix, which is similar to [17]. "I-ICI" finds the inversion of the ICI matrix, and "J-ICI" utilizes Jacobi iterative method.

4.1.2. Comparison between Proposed Method and Resampling Method. In this section, we compare the proposed method

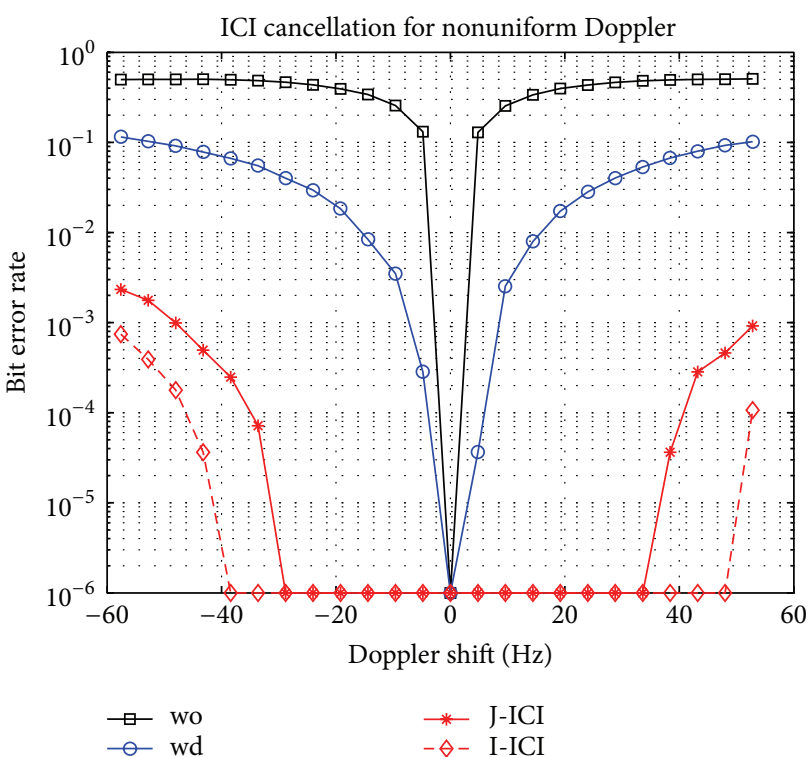

FIGURE 5: Performance of ICI cancellation for nonuniform Doppler.

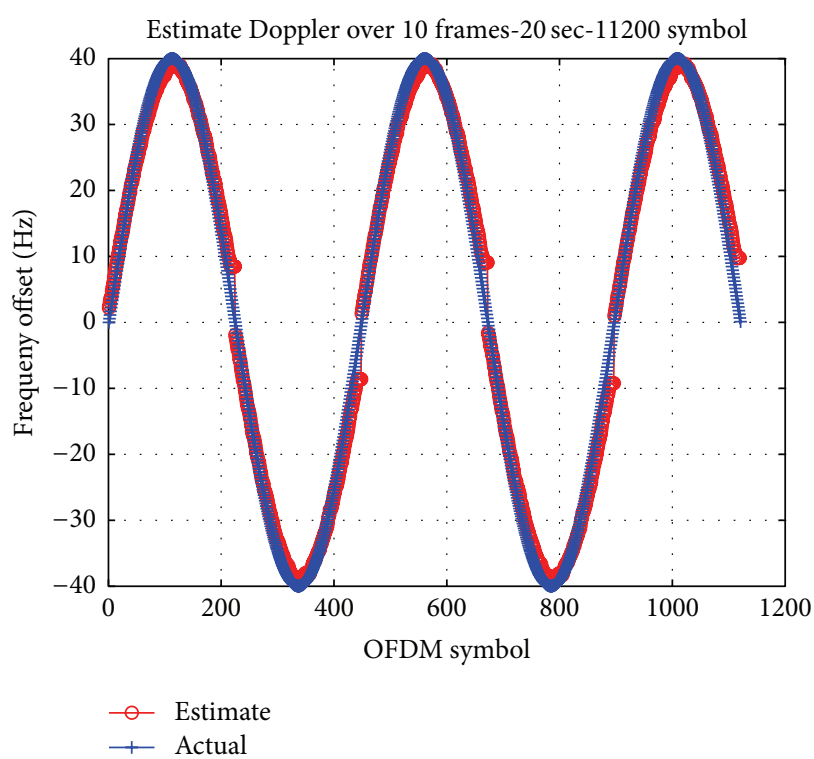

FIGURE 6: Simulation of Doppler estimation for both 16QAM and 64QAM.

against a typical resampling method in [11]. In short, the resampling method resamples the whole data frame of many OFDM symbols with the same scale, then a phase derotation is employed to compensate the residual Doppler. The resampling method utilizes LFM (Linear Frequency Modulation) pre-/post-ample for Doppler rate estimation and must store an entire data frame to estimate the Doppler rate.

Table 3 shows a channel model used in our simulation for two cases. In the first case, the maximum velocity is $2.5 \mathrm{~m} / \mathrm{s}$ which causes a Doppler shift of $40 \mathrm{~Hz}$ and changes over time as shown in Figure 6 for both 16QAM and 64QAM. The 
TABLE 2: Channel for simulation $(\mathrm{CNR}=35 \mathrm{~dB})$.

\begin{tabular}{|c|c|c|c|c|c|c|}
\hline Path & Delay (ms) & Power $(\mathrm{dB})$ & \multicolumn{2}{|c|}{ Doppler rate $\Delta$} & \multicolumn{2}{|c|}{ Doppler shift $\Delta f_{c}(\mathrm{~Hz})$} \\
\hline 1 & 0 & 0 & {$\left[-2.4 \cdot 10^{-3}\right.$} & $\left.2.4 \cdot 10^{-3}\right]$ & {$[-57.6$} & $57.6]$ \\
\hline 2 & 2 & -5 & {$\left[-2.4 \cdot 10^{-3}\right.$} & $\left.2.4 \cdot 10^{-3}\right]$ & {$[-57.6$} & $57.6]$ \\
\hline 3 & 4 & -7 & {$\left[-2.4 \cdot 10^{-3}\right.$} & $\left.2.4 \cdot 10^{-3}\right]$ & {$[-57.6$} & $57.6]$ \\
\hline
\end{tabular}

TABLE 3: Channel for simulation $(\mathrm{CNR}=23 \mathrm{~dB})$.

\begin{tabular}{|c|c|c|c|c|}
\hline Path & Delay (ms) & Power $(\mathrm{dB})$ & Doppler rate & Doppler shift $(\mathrm{Hz})$ \\
\hline 1 & 0 & 0 & {$\left[\begin{array}{ll}-1.6 \cdot 10^{-3} & 1.6 \cdot 10^{-3}\end{array}\right]$} & {$\left[\begin{array}{ll}0 & 40 \mathrm{~Hz}\end{array}\right]$} \\
\hline 2 & 2 & -5 & {$\left[\begin{array}{ll}-1.6 \cdot 10^{-3} & 1.6 \cdot 10^{-3}\end{array}\right]$} & {$\left[\begin{array}{ll}0 & 40 \mathrm{~Hz}\end{array}\right]$} \\
\hline 3 & 4 & -7 & {$\left[\begin{array}{ll}-1.6 \cdot 10^{-3} & 1.6 \cdot 10^{-3}\end{array}\right]$} & {$\left[\begin{array}{ll}0 & 40 \mathrm{~Hz}\end{array}\right]$} \\
\hline
\end{tabular}

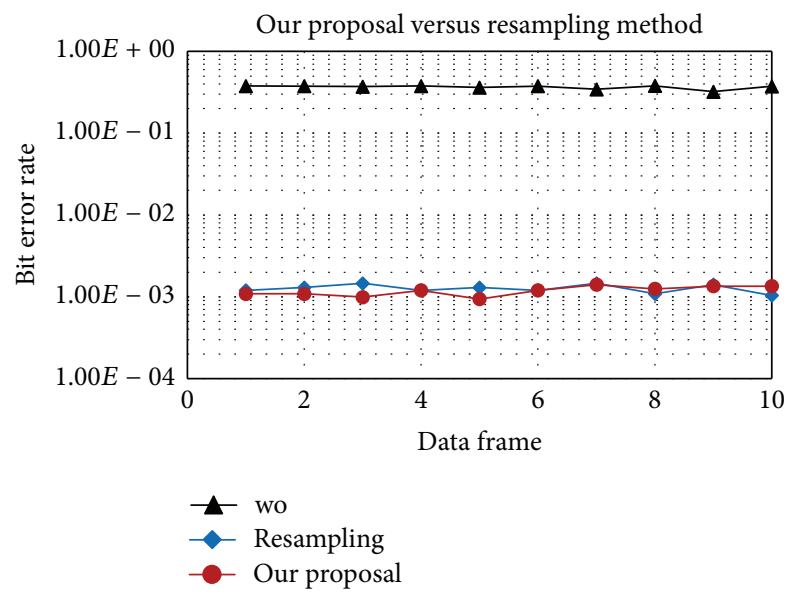

FIGURE 7: 16QAM, proposed method versus the resampling based method.

velocity increases from zero to $2.5 \mathrm{~m} / \mathrm{s}$ with an acceleration rate around $1 \mathrm{~m} / \mathrm{s} / \mathrm{s}$ which is reasonable when considering the fluctuation of transmitters due to ocean waves, or pulling/pushing transmitters in our experiments. As shown in Figure 6, though the maximum Doppler shift as $40 \mathrm{~Hz}$ is greater than $f_{0} / 3$, our tracking method using continual pilots in conjunction with monitoring PDF works well.

In Figures 7 and 8, "wo" means without Doppler compensation. As mentioned before, resampling an entire data frame with a single factor does not fully compensate the time varying Doppler. After resampling, the residual positiondependent Doppler still degrades performance. In contrast, our proposal can track and compensate the Doppler shift symbol-by-symbol, provides a stable performance, and outperforms the resampling method as shown in Figure 8. In detail, with the same acceleration rate of $1 \mathrm{~m} / \mathrm{s} / \mathrm{s}$, after resampling the residual position-dependent Doppler can be negligible for 16QAM and, however, quite significant for 64QAM. Therefore, symbol-by-symbol Doppler compensation provides gain for 64QAM as in Figure 8.

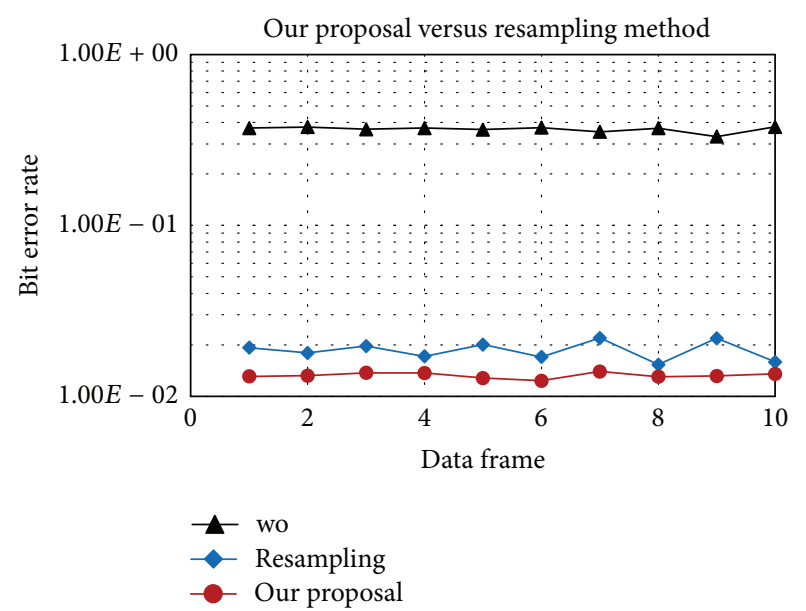

FIGURE 8: 64QAM, proposed method versus the resampling based method.

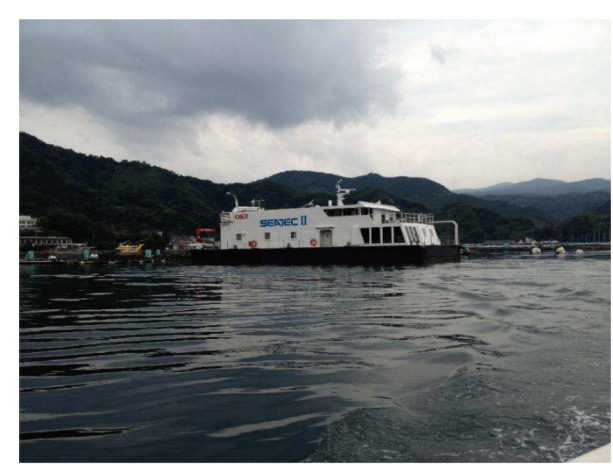

FIGURE 9: The barge.

4.2. Experimental Result. Experiments were conducted inside a barrage in January 2015, in Shizuoka, Japan. There is a moon pool inside the barge as shown in Figures 9 and 10 so that transducers can be dragged down into the water. By a controllable electric motor, a transmitter was periodicity 


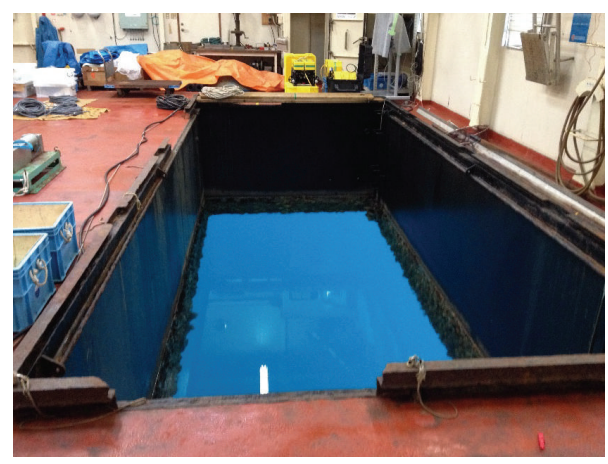

FIgURE 10: The moon pool inside the barge.

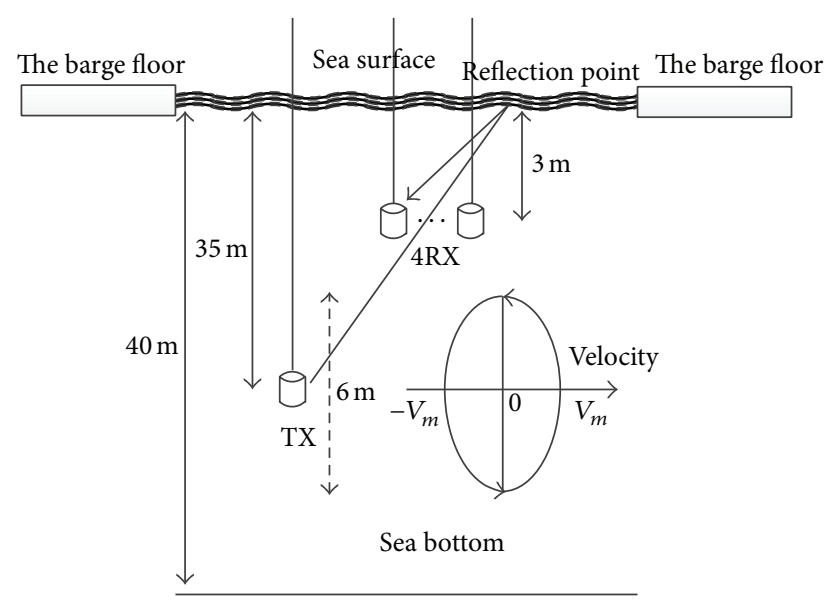

FIGURE 11: The experiment setting.

pulled toward the surface and then pushed toward the bottom as in Figure 11. When approaching the surface or the bottom, the velocity of the transmitter decreases gradually, changes direction, and increases again. So the Doppler shift changes roughly over OFDM symbols in Figures 13 and 14. At the receiver side, four transducers are fixed at $3 \mathrm{~m}$ depth from the surface, and those transducers are spaced equally $10 \mathrm{~cm}$ apart from each other. This space is greater than the wavelength of acoustic signal in our cases, so the received signal at four transducers is uncorrelated and provides diversity gain.

The estimated Delay Profile in Figure 12 is consistent with the experiment setting. In the delay profile, there is a direct path and a surface reflection path, and the distance between them is about $5 \mathrm{~ms}$ which is equivalent to $7.5 \mathrm{~m}$ distance. As the experiment setting, the receivers are at $3 \mathrm{~m}$ depth, and so a reflection path can appear as in Figure 11.

Figures 13 and 14 show the estimation of the time varying Doppler shift over 6 frames, with 16QAM and 64QAM, respectively. The maximum Doppler shift estimated is about $30 \mathrm{~Hz}$ corresponding to the velocity of $2 \mathrm{~m} / \mathrm{s}$, which is consistent with the experiment setting. BER (Bit Error Rate)

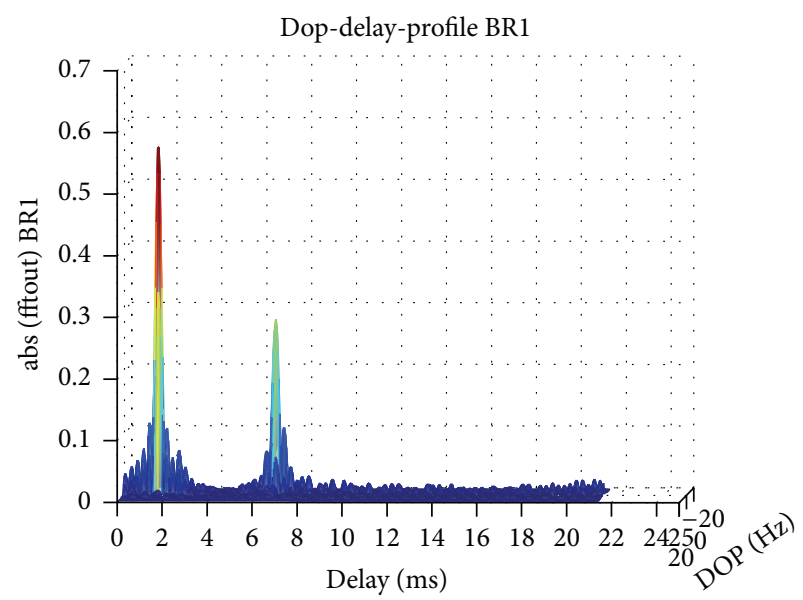

Figure 12: Observed power delay profile.

over frames of 16QAM and 64QAM is shown in Figures 15 and 16, respectively. For both 16QAM and 64QAM cases, during frames 2,3 , and 4 , the Doppler shift is nearly constant over a frame, so our method and the resampling method achieved quite similar performance. With frames 1,5 , and 6 , the velocity changes linearly over a data frame, and our proposal slightly outperformed the resampling method. The performance gain is very small due to the Doppler shift is still small, and the acceleration rate also is small. Similar to simulation result, the performance gain for 64QAM is greater than for 16QAM.

\section{Conclusion}

We presented a transceiver architecture for underwater wireless communication using acoustic OFDM. The highlights are estimation of time varying Doppler through monitoring the drift of PDF combined using continual pilots, and symbolby-symbol Doppler compensation through an ICI matrix for nonuniform Doppler compensation. The simulation and experimental results demonstrate the proposal system provided a stable performance in terms of BER when Doppler changes in time. Overall, a data throughput of $7.5 \mathrm{Kbit} / \mathrm{sec}$ with free-error after Turbo decoding is achieved with 16QAM and 64QAM.

\section{Conflict of Interests}

The authors declare that there is no conflict of interests regarding the publication of this paper.

\section{Acknowledgment}

This study has been carried out as part of the Strategic Information and Communications R\&D Promotion Program (SCOPE) Project of the Ministry of Internal Affairs and Communications Japan. 


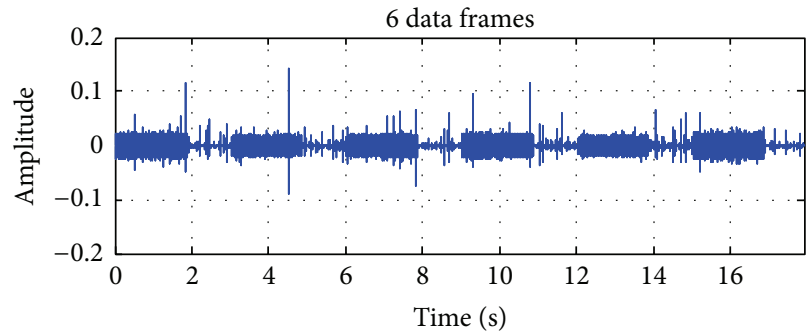

(a)

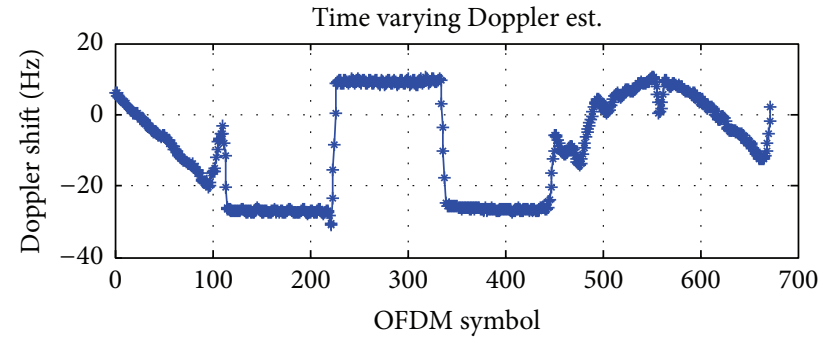

(b)

FIGURE 13: 16QAM, Doppler estimation in the experiments.

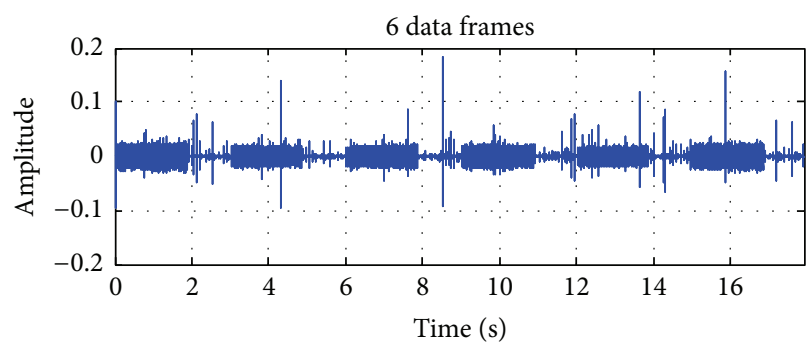

(a)

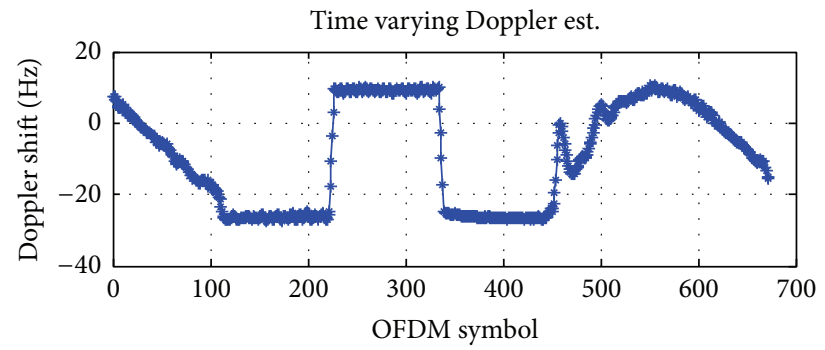

(b)

FIGURE 14: 64QAM, Doppler estimation at the experiment.

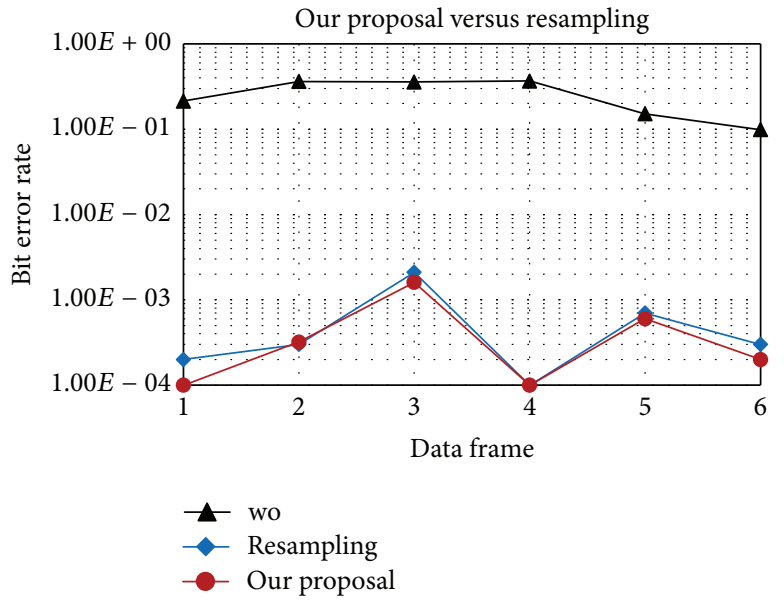

FIGURE 15: 16QAM, BER of proposed method versus the resampling based method in the experiments.

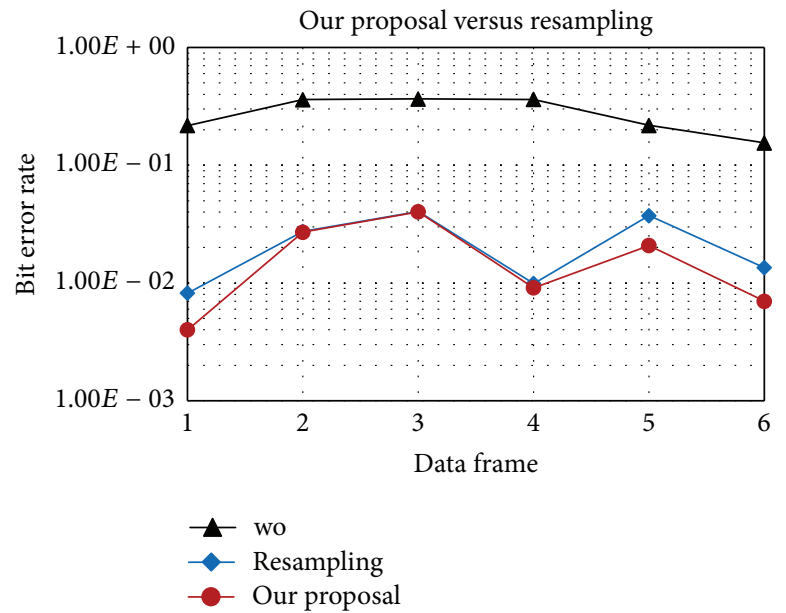

FIGURE 16: 64QAM, BER of proposed method versus the resampling based method in the experiments. 


\section{References}

[1] G. Leus, "On the estimation of rapidly time-varying channels," in Proceedings of the 12th European Signal Processing Conference (EUSIPCO '04), Vienna, Austria, September 2004.

[2] T. Hrycak, S. Das, G. Matz, and H. G. Feichtinger, "Low complexity equalization for doubly selective channels modeled by a basis expansion," IEEE Transactions on Signal Processing, vol. 58, no. 11, pp. 5706-5719, 2010.

[3] V. Fischer, A. Kurpiers, and D. Karsunke, "Ici reduction method for ofdm systems," in Proceedings of the 8th International OFDM-Workshop, pp. 1-5, Hamburg, Germany, September 2003.

[4] Y. Mostofi and D. C. Cox, "ICI mitigation for pilot-aided OFDM mobile systems," IEEE Transactions on Wireless Communications, vol. 4, no. 2, pp. 765-774, 2005.

[5] H. M. Tran and T. Wada, "On improvement of the ici canceller for ofdm mobile dtv receiver," in Proceedings of the 15th International Conference on Advanced Communication Technology (ICACT '13), pp. 1134-1139, PyeongChang, South Korea, January 2013.

[6] B. S. Sharif, J. Neasham, O. R. Hinton, A. E. Adams, and J. Davies, "Adaptive Doppler compensation for coherent acoustic communication," IEE Proceedings: Radar, Sonar and Navigation, vol. 147, no. 5, pp. 239-246, 2000.

[7] B. S. Sharif, J. Neasham, O. R. Hinton, and A. E. Adams, "Computationally efficient Doppler compensation system for underwater acoustic communications," IEEE Journal of Oceanic Engineering, vol. 25, no. 1, pp. 52-61, 2000.

[8] A. E. Abdelkareem, B. S. Sharif, C. C. Tsimenidis, and J. A. Neasham, "Time varying Doppler-shift compensation for ofdm-based shallow underwater acoustic communication systems," in Proceedings of the 8th IEEE International Conference on Mobile Ad-Hoc and Sensor Systems (MASS '11), pp. 885-891, IEEE, Valencia, Spain, October 2011.

[9] A. Abdelkareem, B. Sharif, C. Tsimenidis, J. Neasham, and O. Hinton, "Low-complexity Doppler compensation for OFDMbased underwater acoustic communication systems," in Proceedings of the IEEE OCEANS 2011, pp. 1-6, Santander, Spain, June 2011.

[10] A. E. Abdelkareem, Doppler compensation algorithms for dspbased implementation of ofdm underwater acoustic communication systems [Ph.D. thesis], Newcastle University, Newcastle, UK, 2012.

[11] B. Li, S. Zhou, M. Stojanovic, L. L. Freitag, and P. Willett, "Multicarrier communication over underwater acoustic channels with nonuniform Doppler shifts," IEEE Journal of Oceanic Engineering, vol. 33, no. 2, pp. 198-209, 2008.

[12] B. Li, S. Zhou, M. Stojanovic, L. Freitag, and P. Willett, "Nonuniform doppler compensation for zero-padded ofdm over fastvarying underwater acoustic channels," in Proceedings of the Europe OCEANS, pp. 1-6, IEEE, Aberdeen, UK, June 2007.

[13] B. Li, J. Huang, S. Zhou et al., "MIMO-OFDM for high-rate underwater acoustic communications," IEEE Journal of Oceanic Engineering, vol. 34, no. 4, pp. 634-644, 2009.

[14] T. M. Hai, Y. Matsuda, T. Suzuki, and T. Wada, "Ultrasonic diversity OFDM transceiver architecture with impulsive noise. Cancelling for shallow sea communication," in Proceedings of the 2nd International Conference and Exhibition on Underwater Acoustic (UA '14), pp. 1227-1232, Rhodes, Greece, June 2014.

[15] R. Saotome, T. M. Hai, Y. Matsuda, T. Suzuki, and T. Wada, "An OFDM receiver with frequency domain diversity combined impulsive noise canceller for underwater network," The Scientific World Journal, vol. 2015, Article ID 841750, 10 pages, 2015.

[16] T. Suzuki, H. M. Tran, and T. Wada, "An underwater acoustic OFDM communication system with shrimp (impulsive) noise cancelling," in Proceedings of the International Conference on Computing, Management and Telecommunications (ComManTel '14), pp. 152-156, IEEE, Da Nang, Vietnam, April 2014.

[17] M. Stojanovic, "Low complexity OFDM detector for underwater acoustic channels," in Proceedings of the IEEE OCEANS 2006, pp. 1-6, Boston, Mass, USA, September 2006. 


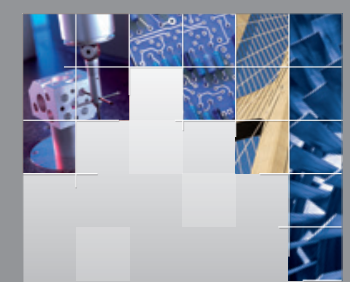

\section{Enfincering}
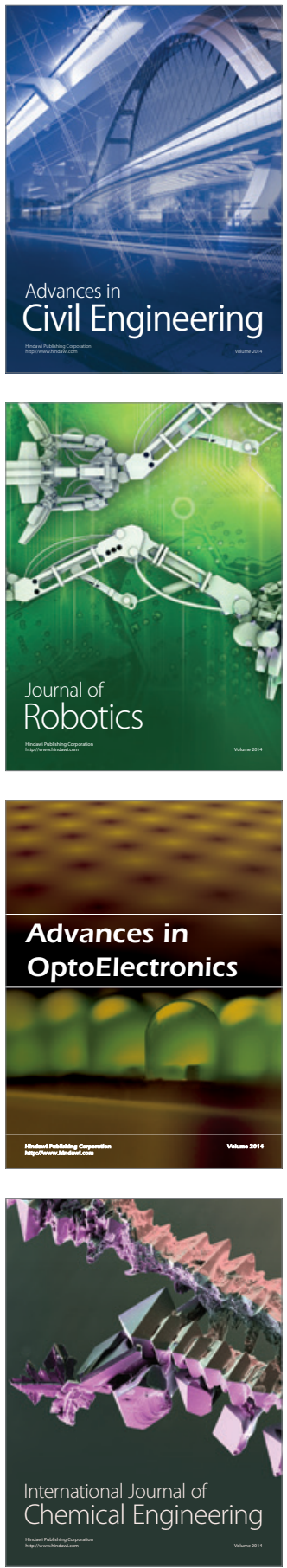

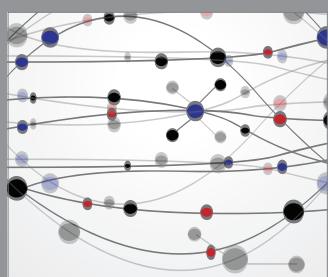

The Scientific World Journal

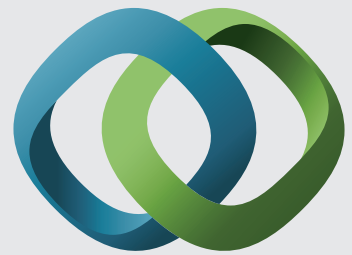

\section{Hindawi}

Submit your manuscripts at

http://www.hindawi.com
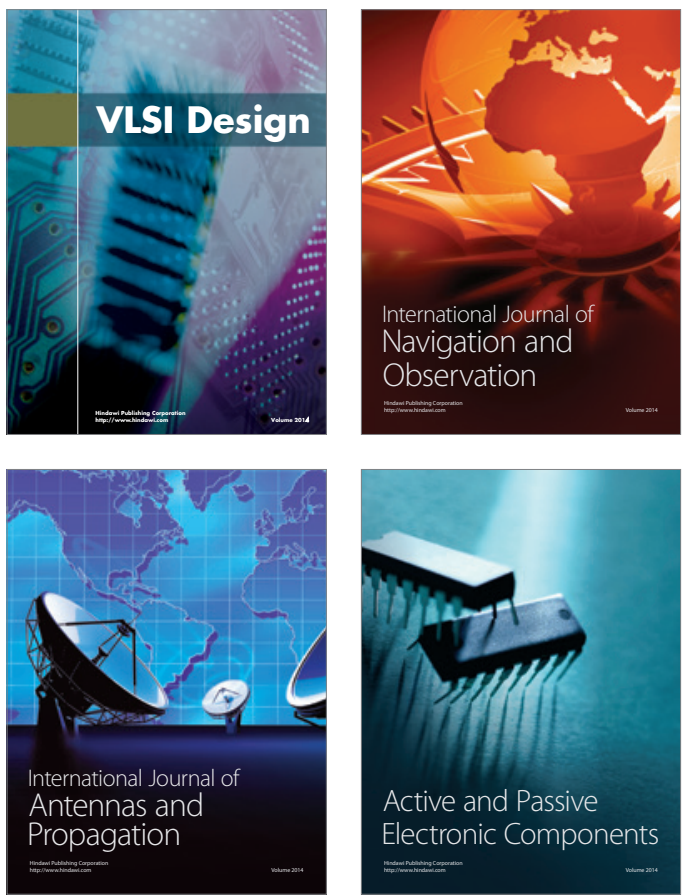
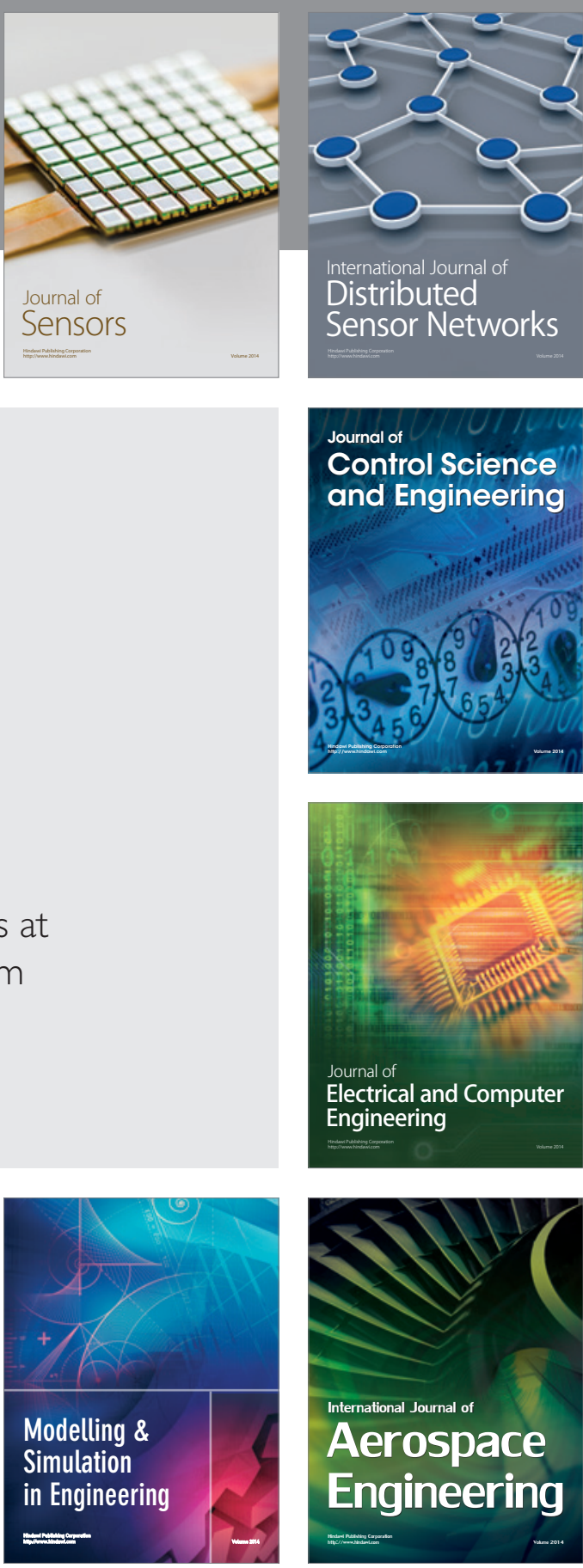

International Journal of

Distributed

Sensor Networks

Journal of

Control Science

and Engineering
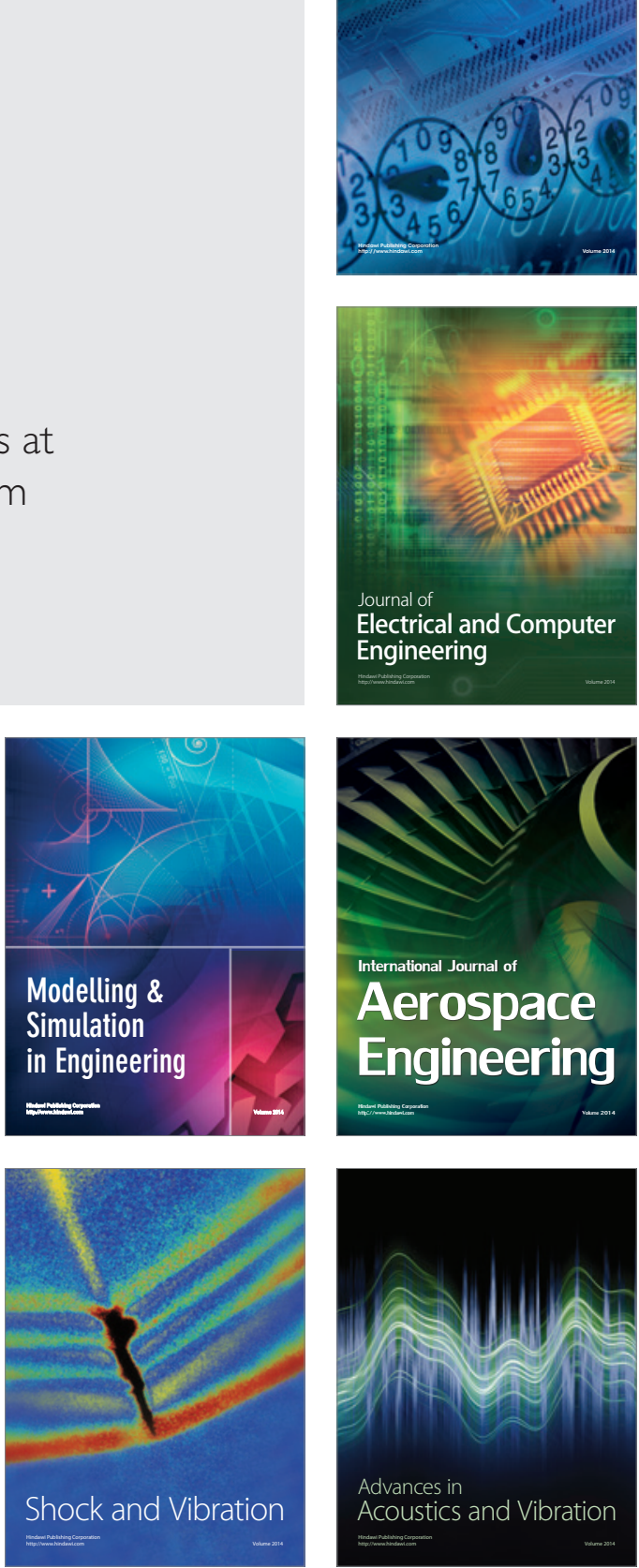